AperTO - Archivio Istituzionale Open Access dell'Università di Torino

\title{
Generating Function Approach to the Calculation of Spectral Band Shapes of Free-Base Chlorin Including Duschinsky and Herzberg-Teller Effects
}

\section{This is the author's manuscript}

Original Citation:

\section{Availability:}

This version is available http://hdl.handle.net/2318/137257

since 2021-09-13T11:55:47Z

Published version:

DOI:10.1021/jp307887s

Terms of use:

Open Access

Anyone can freely access the full text of works made available as "Open Access". Works made available under a Creative Commons license can be used according to the terms and conditions of said license. Use of all other works requires consent of the right holder (author or publisher) if not exempted from copyright protection by the applicable law. 


\title{
A generating function approach to the calculation of
}

\section{spectral band shapes of free-base chlorin including}

\section{Duschinsky and Herzberg-Teller effects}

\author{
Raffaele Borrelli, ${ }^{*}, \dagger$ Amedeo Capobianco, ${ }^{\ddagger}$ and Andrea Peluso ${ }^{*}, \ddagger$ \\ Dipartimento di Science Agrarie, Forestali e Alimentari, Università di Torino, I-10095 \\ Grugliasco (TO), Italy, and Dipartimento di Chimica e Biologia, Università di Salerno, I-84084, \\ Fisciano (SA), Italy \\ E-mail: raffaele.borrelli@unito.it; apeluso@unisa.it
}

${ }^{*}$ To whom correspondence should be addressed

${ }^{\dagger}$ Dipartimento di Science Agrarie, Forestali e Alimentari, Università di Torino, I-10095 Grugliasco (TO), Italy

†Dipartimento di Chimica e Biologia, Università di Salerno, I-84084, Fisciano (SA), Italy 


\begin{abstract}
A generating function approach to the calculation of spectral band shapes including Duschinsky and Herzberg-Teller effects is proposed and applied to the computation of the free-base chlorin Q absorption bands, using molecular geometries and normal vibrations obtained by density functional theory computations. The results clearly show that non-Condon effects can significantly affect the relative intensities of the weakest $\mathrm{Q}_{y}$ and, to a lesser extent, $\mathrm{Q}_{x}$ bands. The proposed approach is extremely powerful and can be used in the cases where the molecular size makes the direct calculation of Franck-Condon integrals by recurrence formulae prohibitive.
\end{abstract}




\section{Introduction}

The calculation and analysis of absorption and emission band shapes of molecules from first principles is a fundamental problem of modern physical chemistry, with a widespread range of applications, extending from the understanding of the basic aspects of light-matter interaction to the development of organic dyes with tailored spectroscopic properties. ${ }^{1-4}$ Apart from the known difficulties in obtaining equilibrium geometries and normal modes of excited states, a problem significantly relieved by the development of time-dependent density functional theory (TDDFT), the direct calculation of Franck-Condon factors, the most common approach for computing band shapes, ${ }^{5-16}$ can pose problems for large size molecules, being based on the use of multi-indices recurrence relations, which require the storage of a large number of FC integrals.

Several ways out have been considered. Algorithms based either on a judicious way of choosing normal mode excitations, ${ }^{13,14,17,18}$ or on dividing the space of vibrational states into subgroups, each of them having a defined number of degrees of freedom allowed to be excited, ${ }^{18}$ have been proposed but, although very useful, they do not provide a general solution. The recently proposed perturbative approach allows to avoid the storage of large sequences of integrals, but the calculation of higher order contributions, needed in the case of strong mode mixing, are difficult to carry out. ${ }^{12}$ Criteria for finding out contributions to the total spectral intensity from specific subsets of FC factors, without explicitly calculating the integrals have also been provided, ${ }^{17}$ but the approach does not guarantee that a converged spectrum can actually be computed, since the number of FC factors can still be too large for a direct calculation.

A different approach is provided by the theory of the generating function (GF) developed by Lax, Kubo and Toyozawa in the fifties. ${ }^{19,20}$ Mukamel $^{21}$ was among the first authors to realize that such an approach is to be preferred in band shape simulations of complex molecular systems, since it bypasses the calculation of FC integrals, being therefore particularly suitable for large size molecules. However the GF approach also poses problems for interpretative purposes, because it does not directly provide the deconvolution of the spectrum into its vibrational components, which has to be done by a posteriori analysis. Other authors have developed such an approach in 
the past, although not explicitly tailored to the computation of molecular electronic spectra ${ }^{22,23}$. More recently Pollak and collaborators ${ }^{24,25}$ have introduced an alternative procedure to develop closed expressions for the generating function in the case of Franck-Condon transtions, and Shuai and coworkers ${ }^{26-29}$ have extended Pollak methodology, including Herzberg-Teller effects. Along that line, Berger and Huh have recently developed a cumulant expansion of the lineshape function including both Duschinsky and non-Condon effects ${ }^{30}$. All these contributions could be very important for the development of new methods and new functionals tailored to the description of electronic excited states of molecules, inasmuch they provide easy to handle and very reliable methods for using band shapes as benchmarks. ${ }^{31}$

In this paper we discuss an approach in which the overall spectral lineshape is computed using the GF technique and the deconvolution into its components, where needed, is provided a posteriori by a direct calculation of FC integrals. The method include both displacements and Duschinsky rotations of normal modes, and Herzberg-Teller (HT) effects. The methodology has been applied to the calculation and analysis of the $\mathrm{Q}_{x}$ and $\mathrm{Q}_{y}$ bands of the free-base chlorin, the precursor of chlorophylls, a large chemical system with 114 vibrational normal modes.

\section{Generating function theory of lineshapes}

In this section we shortly illustrate the GF approach, deriving an easy to handle formulation which includes Herzberg-Teller contribution.

The cross section for a radiative transition from a manifold of thermally populated vibronic states $\left|v_{g}\right\rangle$ to a manifold of vibronic states $\left|v_{e}\right\rangle$ is:

$$
I(\omega, T)=\left(\hbar Z_{v}\right)^{-1} \sum_{\alpha=x, y, z v_{g}, v_{e}} e^{-\beta E\left(v_{g}\right)}\left|\left\langle v_{g}\left|\mu_{\alpha}^{g e}\right| v_{e}\right\rangle\right|^{2} \delta\left(\omega+E\left(v_{g}\right)-E\left(v_{e}\right)-\Delta E_{e g}\right)
$$

where $E\left(v_{g}\right)$ and $E\left(v_{e}\right)$ are the energies of the vibrational states in the initial and final electronic state, $|g\rangle$ and $|e\rangle, \Delta E_{e g}$ is the electronic energy difference, $\mu_{\alpha}^{g e}$ is the $\alpha$-th component of the electric transition dipole moment, and $\beta=1 / k T$, with $k$ the Boltzmann constant. 
Including first order HT effects, the electric transition dipole moment can be expressed as a power expansion with respect to the normal coordinates of the initial state $\mathbf{Q}_{g}$ about the equilibrium position:

$$
\mu_{\alpha}^{g e}=\mu_{\alpha}^{\circ g e}+\sum_{j}\left(\frac{\partial \mu_{\alpha}^{g e}}{\partial Q_{g j}}\right)_{0} Q_{g j} \quad \alpha=x, y, z
$$

The $\mathbf{Q}_{g}$ vector is related to his counterpart of the final electronic state $\mathbf{Q}_{e}$ by the Duschinsky transformation:

$$
\mathbf{Q}_{e}=\mathbf{J} \mathbf{Q}_{g}+\mathbf{K}
$$

where $\mathbf{J}$ is the normal mode rotation matrix and $\mathbf{K}$ the displacement vector. ${ }^{32}$

Following the seminal works of $\operatorname{Lax}^{19}$ and Kubo, ${ }^{33}$ the spectral distribution $I(\omega, T)$ can be conveniently rewritten as:

$$
I(\omega, T)=2 \pi \int_{-\infty}^{+\infty} e^{-i\left(\omega+\Delta E_{e g}\right) \tau} f(\tau) d \tau
$$

where

$$
f(\tau)=\operatorname{Tr}\left\{\mu e^{-i \tau H_{e}} \mu e^{-(\beta-i \tau) H_{g}}\right\} / \operatorname{Tr}\left\{e^{-\beta H_{g}}\right\}
$$

and $H_{g}, H_{e}$ are the Hamiltonian operators of the initial (ground) and final (excited) electronic states respectively. If the latter ones are expressed in harmonic approximation, the correlation function $f(\tau)$ assumes the following closed form (see Supporting Information): ${ }^{33}$

$$
f(\tau)=[\operatorname{det} \Phi]^{-1 / 2} \exp \left[-\tilde{\Delta} \mathbf{T}_{g}\left(\mathbf{T}_{g}+\mathbf{T}_{e}\right)^{-1} \mathbf{T}_{e} \Delta\right] \cdot g_{\mu}(\tau)
$$


where

$$
\begin{aligned}
& \mathbf{T}_{g}=\omega_{g} \tanh \left[(\beta-i \tau) \omega_{g} / 2\right] ; \quad \mathbf{T}_{e}=\tilde{\mathbf{J}} \omega_{e} \tanh \left(i \tau \omega_{e} / 2\right) \mathbf{J} \\
& \mathbf{C}_{g}=\omega_{g} / \tanh \left[(\beta-i \tau) \omega_{g} / 2\right] ; \quad \mathbf{C}_{e}=\tilde{\mathbf{J}} \omega_{e} / \tanh \left(i \tau \omega_{e} / 2\right) \mathbf{J} \\
& \Phi=\left[2 \sinh \left(\beta \omega_{g} / 2\right)\right]^{-2} \omega_{g}^{-1} \sinh \left[(\beta-i \tau) \omega_{g}\right]\left(\mathbf{T}_{g}+\mathbf{T}_{e}\right)\left(\mathbf{C}_{g}+\mathbf{C}_{e}\right) \omega_{e}^{-1} \sinh \left(i \tau \omega_{e}\right) \\
& \Delta=-\tilde{\mathbf{J}} \mathbf{K}
\end{aligned}
$$

and

$$
g_{\mu}(\tau)=\left|\mu^{\circ}\right|^{2}+2 \sum_{\alpha, r} \mu_{\alpha}^{\circ} \mu_{\alpha r}^{(1)} D_{r}+\sum_{\alpha, r, s} \mu_{\alpha r}^{(1)} A_{r s} \mu_{\alpha s}^{(1)}
$$

with

$$
\begin{aligned}
\mu_{\alpha r}^{(1)} & =\left(\frac{\partial \mu_{\alpha}^{g e}}{\partial Q_{g r}}\right)_{0} \\
\mathbf{X} & =\mathbf{T}_{e}+\mathbf{T}_{g} ; \quad \mathbf{Y}=\mathbf{C}_{e}+\mathbf{C}_{g} \\
D_{r} & =\left(\mathbf{X}^{-1} \mathbf{T}_{e} \Delta\right)_{r} ; \quad A_{r s}=\left(\mathbf{X}^{-1}-\mathbf{Y}^{-1}\right)_{r s} / 2+D_{r} D_{s} .
\end{aligned}
$$

It is easy to see that in the above formulation the molecular lineshape has three contributions: i) pure FC transitions, ii) pure HT transitions and iii) transitions deriving from FC-HT intereferences, ruling out the possibility to separately analyse the contribution of the two type of transitions to the overall spectrum.

It is worth mentioning that Shuai and co-workers have recently derived an equivalent expression for molecular lineshapes based on the GF formalism, but using a different mathematical approach. ${ }^{28}$ Our methodology differs from their one inasmuch it requires the inversion of $N \times N$ matrices.

The GF formulation is very efficient for numerical treatments because the calculation of $I(\omega, T)$ 
can be recast into a discrete Fourier transform problem

$$
I(\omega, T)=\frac{1}{N} \sum_{k=1}^{N} w\left(\tau_{k}\right) f\left(\tau_{k} ; T\right) e^{i \omega \tau_{k}}
$$

where $w(\tau)$ is a proper window function, necessary to avoid boundary and spectral leakage problems. ${ }^{34}$

The appealing feature of the GF approach is in its ability to easily handle the calculation of the temperature dependence of electronic band shapes, since, at variance with the direct evaluation of Eq. (1), its computational cost is independent of the value of the temperature. As main limitation, the method does not provide the assignment of the simulated spectrum in terms of its vibronic components, requiring for that an a posteriori analysis.

\section{Computational details}

The ground state equilibrium geometry and the normal modes of vibration of the free-base chlorin have been computed using density functional theory (DFT), employing the hybrid functional PBE0 and the standard 6-31+G(d,p) basis set. This functional has already proved to provide good results for the excited state geometry and transition moment derivative of porphin. ${ }^{18}$ Equilibrium geometries and normal modes of the two lowest excited states have been calculated by the timedependent density functional theory (TD-DFT) approach, using the same functional and basis set of the ground state calculation.

The derivatives of the transiton dipole moment $\mu_{\alpha}^{g e}$ at the equilibrium position, with respect to the Cartesian coordinates $\xi_{i}=\left(x_{i}, y_{i}, z_{i}\right)$ of the $i$-th atom, have been calculated by the second order formula:

$$
\left(\frac{\partial \mu_{\alpha}^{g e}}{\partial \xi_{i}}\right)_{\xi^{\circ}} \approx\left(\mu_{\alpha}^{g e}\left(\xi_{i}^{\circ}+\Delta \xi_{i}\right)-\mu_{\alpha}^{g e}\left(\xi_{i}^{\circ}-\Delta \xi_{i}\right)\right) /\left(2 \Delta \xi_{i}\right), \quad \alpha=x, y, z
$$


and then projected along the normal coordinates:

$$
\left(\frac{\partial \mu_{\alpha}^{g e}}{\partial Q_{k}}\right)_{0}=\sum_{i}\left(M^{-1 / 2} \mathscr{L}\right)_{i k}\left(\frac{\partial \mu_{\alpha}^{g e}}{\partial \xi_{i}}\right)_{\xi^{\circ}}, \quad \alpha=x, y, z
$$

where $M$ is the usual $3 N \times 3 N$ diagonal matrix of atomic masses, and $\mathscr{L}$ is the $3 N \times 3 N-6$ matrix of normal vibrations expressed in terms of mass-weighted Cartesian displacements.

All calculations have been peformed using the Gaussian09 software package ${ }^{35}$; the differentiation step $\Delta \xi$ has been set to $0.05 \AA$. Noteworthy, the transition moment at the $6 N$ displaced geometries can be obtained by Gaussian09 as a by-product of the numerical calculation of the excited state Hessian matrix.

The Duschinsky matrix $\mathbf{J}$ and the displacement vector $\mathbf{K}$ have been computed using the standard procedure. ${ }^{10,32,36-38}$ The generating function approach has been implemented in a locally modified version of the MolFC software ${ }^{39}$.

The spectral lineshapes have been calculated using $2^{13}$ time steps, achieving a final resolution

of $1 \mathrm{~cm}^{-1}$; a four-term Blackman-Harris window has been used. The assignment of the vibronic peaks to a specific transition is performed by first computing the entire lineshape, then searching for possible vibronic states in the selected energy range, and computing the associated FC integrals. A back-tracking algorithm ${ }^{40}$ has been implemented for the state search.

\section{Vibronic structure of the $Q$ bands of free-base chlorin}

Chlorin, Figure 1, is the porphin derivative obtained by the addition of two hydrogen atoms across one of the two semi-isolated double bonds of a pyrrole ring without an inner proton. The chemical reduction does not substantially alter the characteristic spectral pattern: as for porphin there are two absorptions, each of them constituted by two electronic transitions with different transition moment polarization: the Soret band in the blue region of the spectrum and the so called Q band in the red one. ${ }^{41,42}$ However, in chlorins the intensities of the two absorptions is considerably different from porphin ones, the Soret band is considerably less intense, whereas the intensity of 
the $\mathrm{Q}$ band is markedly increased, being responsible for the green colors which characterize many of chlorins. Noteworthy, the intensity decrease of the Soret band is almost equally balanced by the increase in absorption of the Q band, supporting the hypothesis that Gouterman's four orbital model also apply to chlorin. ${ }^{43}$ DFT computations supports the above hypothesis. The computed energy transitions for the lowest energy excited states, together with oscillator strengths, are reported in Table 1 for both the gas-phase and the n-octane solution. Denoting by $\mathrm{H}$ and L the HOMO and LUMO, respectively, the $\mathrm{Q}_{x}$ and $\mathrm{Q}_{y}$ transition are given by:

$$
\begin{aligned}
& \mathrm{Q}_{x}=-0.37(\mathrm{H}-1 \rightarrow \mathrm{L}+1)+0.61(\mathrm{H} \rightarrow \mathrm{L}) \\
& \mathrm{Q}_{y}=0.55(\mathrm{H}-1 \rightarrow \mathrm{L})+0.45(\mathrm{H} \rightarrow \mathrm{L}+1)
\end{aligned}
$$

in full agreement with Gouterman's four orbital model. ${ }^{41}$ As concerns the two Soret bands, DFT computations predict the involvement of a lowest energy $\pi$ orbital HOMO-2, fully localized on the unsaturated pyrrole ring:

$$
\begin{gathered}
\mathrm{B}_{x}=0.56(\mathrm{H}-1 \rightarrow \mathrm{L}+1)+0.34(\mathrm{H} \rightarrow \mathrm{L})+0.27(\mathrm{H}-2 \rightarrow \mathrm{L}+1) \\
\mathrm{B}_{y}=-0.32(\mathrm{H}-1 \rightarrow \mathrm{L})+0.38(\mathrm{H} \rightarrow \mathrm{L}+1)+0.51(\mathrm{H}-2 \rightarrow \mathrm{L})
\end{gathered}
$$

The computed transition energies, reported in are slightly overestimated, ca. $0.2-0.3 \mathrm{eV}$ for the $\mathrm{Q}$ bands and $0.4 \mathrm{eV}$ for the Soret ones, . The use of a larger, triple zeta basis set, with diffuse functions on both hydrogen and heavier atoms, does not significantly improve the results.

The room temperature absorption spectrum of free-base chlorin $\left(\mathrm{H}_{2} \mathrm{Ch}\right)$ in n-octane shows in the visible region a comparatively stronger band at $636 \mathrm{~nm}\left(15710 \mathrm{~cm}^{-1}\right)$, which is generally agreed to be the origin of the lowest energy $\mathrm{Q}_{x}$ transition, and a second much weaker absorption band, located at ca. $490 \mathrm{~nm}$. While the former transition exhibits a well resolved vibrational structure, extending up to $570 \mathrm{~nm}\left(17500 \mathrm{~cm}^{-1}\right)$, the latter one appears almost as a broad unstructured absorption, consisting of only two peaks at 20400 and $20800 \mathrm{~cm}^{-1}$, neither of which, on the basis of magnetic circular dichroism and fluorescence polarization spectra, ${ }^{44,45}$ is believed to be the origin of the $\mathrm{Q}_{y}$ transition. 
DFT computations predict that the equilibrium geometries of the first two excited states are both very similar to the ground state one. The root mean square variation of the whole set of inter-atomic distances is $0.023 \AA$ for both $\mathrm{Q}_{x}\left(\mathrm{~S}_{1}\right)$ and $\mathrm{Q}_{y}\left(\mathrm{~S}_{2}\right)$ with respect to the ground state, with maximum differences of $0.080 \AA$ for the former and $0.077 \AA$ for the latter. In both excited states $\mathrm{C}_{2 v}$ symmetry is lost: the molecule is not planar, due to an out of plane distortion of the saturated $\mathrm{C}-\mathrm{C}$ bonds, in which the two carbon atoms are shifted up and down the molecular plane. Since the distortion in one or the opposite direction are symmetric, both electronic states exhibit a double well potential energy profile along that coordinate, the barrier to inversion being so small $(0.002 \mathrm{kcal} / \mathrm{mol}$ at $\mathrm{PBE} 0 / 6-31+\mathrm{G}(\mathrm{d}, \mathrm{p})$ level $)$ that the effect of the double well potential can be safely neglected.

The parameters which mostly determine the intensities of the vibronic transitions, i.e. normal mode equilibrium position displacements and the first derivatives of the electric dipole transition moments with respect to the normal coordinates, have been reported in Table 2 and Table 3 , for the most active normal modes of the $\mathrm{Q}_{x} \leftarrow \mathrm{S}_{0}$ and $\mathrm{Q}_{y} \leftarrow \mathrm{S}_{0}$ transitions, respectively.

The high resolution absorption spectrum of chlorin has been recorded as an excitation spectrum in n-octane crystal at $5 \mathrm{~K} .{ }^{46}$ The excitation spectrum in the region of the $\mathrm{Q}_{x}$ transition consists of a very narrow origin, with three clearcut peaks at 154, 288 and $305 \mathrm{~cm}^{-1}$ from the origin. Then, there are other clearly distinguishable peaks at 711,870 and $939 \mathrm{~cm}^{-1}$, followed by a weak but extended vibronic activity in the range $1200-1600 \mathrm{~cm}^{-1}$. The spectrum computed by considering only FC factors and that including both FC and HT contributions are reported in Figure 2 and Figure 3, respectively. First of all, there are not significant HT contributions for the $\mathrm{Q}_{x}$ band, the FC and FCHT spectra are quite similar, as expected because the computed transition dipole moment at the $\mathrm{S}_{0}$ equilibrium geometry is 1.26 a.u. while the transition moment derivatives with respect to normal modes are, on average, at least one order of magnitude lower, see Table 2. Only for a couple of vibrational modes, one at $1417 \mathrm{~cm}^{-1}$ (mode 82) the other at $1625 \mathrm{~cm}^{-1}$ (mode 90), the transition dipole moment derivatives are comparatively higher (see Table 2), the latter one, being not a displaced mode, exhibits a distinguishable HT activity toghether with other vibrations 
arount $1400 \mathrm{~cm}^{-1}$ (see Figure 3).

The computed spectrum qualitatively reproduce all the main features of the observed one, a quantitative comparison being ruled out by the fact that during excitation free-base chlorin is burned into its photoproducts, so that experimental intensities have to be taken as approximate ones. ${ }^{46}$ The most intense transition is predicted to be at ca. $20 \mathrm{~cm}^{-1}$, because of the significantly displacement of the lowest frequency mode. Next, two narrow progressions in the range 130-210 $\mathrm{cm}^{-1}$ and 270-410, with two most intense peaks at 315 and $331 \mathrm{~cm}^{-1}$, and other weak progressions, peaked at 750 and 1100,1410 , and $1650 \mathrm{~cm}^{-1}$, are predicted, in qualitative agreement with the observed spectrum. Lastly, a comparatively stronger progression, due to the significant displacement of the mode at $3077 \mathrm{~cm}^{-1}$, is predicted at $3125 \mathrm{~cm}^{-1}$, a region in which the experimental spectrum also shows some vibronic activity.

Almost all the signals observed in the $\mathrm{Q}_{x}$ region of the excitation spectrum can be traced back to vibrational modes whose equilibrium positions is displaced in the transition, see Table 2, and HT effects contributes to changing their relative intensities. The most displaced mode is the lowest frequency one; short progressions due to excitation of this mode are observed in the computed spectrum for all the higher frequency signals. Furthermore, displaced modes are predicted at approximately $150,300,730 \mathrm{~cm}^{-1}$, regions in which even the room temperature spectrum shows weak peaks, and at 1000,1300 , and $1450 \mathrm{~cm}^{-1}$, where the low temperature spectrum shows an important vibronic activity.

For the $\mathrm{Q}_{y}$ transition the situation is quite different since the number of displaced modes is similar to that of the $\mathrm{Q}_{x}$ transition, but the HT terms become extremely important. Inspection of Table 3 shows that there are three modes whose equilibrium position is significantly displaced, the lowest energy mode, which is fully localized on the unsaturated pyrrole ring, an expansion/compression mode involving the whole macrocycle, and an in plane bending mode involving the exocyclic hydrogen atoms, predicted at $305 \mathrm{~cm}^{-1}$ and $1387 \mathrm{~cm}^{-1}$, respectively. By contrast, strong normal mode rotations are present. By considering only mixing coefficient above 0.4 , most of the modes in the range $850-1650 \mathrm{~cm}^{-1}$ are strongly mixed each other. As well known Duschinsky mixing 
is a second order effect, ${ }^{12}$ which can be important in regulating interference effects between FC and FCHT contributions, without giving rise to a direct vibronic activity. Accordingly, although DFT computations predict a significant mode mixing, the computed Franck-Condon spectrum of the $\mathrm{Q}_{y} \leftarrow \mathrm{S}_{0}$ transition of chlorin at $\mathrm{T}=5 \mathrm{~K}$, shown in Figure 4, is dominated by the 0-0 band, apart that sharp transition, there are a few weak transitions, due to the excitation of the displaced modes at $305 \mathrm{~cm}^{-1}, 1078$ and $1387 \mathrm{~cm}^{-1}$, see Table 3 .

Herzberg-Teller effects completely change the shape of the computed spectrum, compare Figure 4 and Figure 5 , as can be easily understood by inspecting Table 3, where the derivatives of the electric transition dipole moment (only the $y$ component) with respect to normal coordinates are reported. The computed transition dipole moment for the $\mathrm{Q}_{y} \leftarrow \mathrm{S}_{0}$ transition is 0.275 a.u., about one order of magnitude less than that for the $\mathrm{Q}_{x} \leftarrow \mathrm{S}_{0}$ transition (1.26 a.u.). There are sixteen modes for which the HT factor is larger than the zero order transition dipole moment by one order of magnitude, and three of them exhibit HT factors larger by two order of magnitudes. The latter modes fall in the region $700-850 \mathrm{~cm}^{-1}$, where the spectrum computed including HT effects shows comparatively intense signals.

The high resolution excitation spectrum recorded in n-octane at $7 \mathrm{~K}$ is very broad in the $\mathrm{Q}_{y}$ region, $20000-21500 \mathrm{~cm}^{-1}$ and consists of several well resolved peaks superposed to a continuum. The broadness and the erratic structure of the excitation spectrum has made it difficult the assignments of the observed peaks; the origin of the $\mathrm{Q}_{y}$ band has not been assigned and the possible involvement of a low lying $\mathrm{n} \pi^{*}$ transition has also been hypothesized to explain some spectral features. ${ }^{46}$ By contrast, the computed spectrum at $7 \mathrm{~K}$, consists of isolated peaks, which, even by convolution using large bandwidths, do not give rise to a broad continuum. Since the $\mathrm{Q}_{x}$ region of the spectrum is well reproduced by computations, testifying about the accuracy of the adopted computational level, we are led to suppose that the broadness of the absorption band is due to electronic couplings with the Soret band, as also suggested by the observed oscillator strengths of chlorin, indicating intensity borrowing from the $\mathrm{B}$ to the $\mathrm{Q}$ band. ${ }^{43}$ Notwithstanding, leaving apart the relative intensities of the spectral signals, which of course cannot be reproduced by a model 
which does not include interactions with other electronic states, the computed HT spectrum allows for a tentative assignment of the most important spectral features of the $\mathrm{Q}_{y}$ transition. The frequency distribution of the computed peaks lead us to assign the series of peaks computed at about $100 \mathrm{~cm}^{-1}$ to the broad signal observed at $3664 \mathrm{~cm}^{-1}$ above the 0-0 $\mathrm{Q}_{x}$ transition (for the sake of simplicity we use the same scheme adopted by Huang et $\mathrm{al}^{46}$, who refers all wavenumbers to the 0-0 $\mathrm{Q}_{x}$ transition). Then the broad signal peaked at 4037 can be reasonably assigned to the two groups of transitions predicted around 300 and $400 \mathrm{~cm}^{-1}$, the former due to the displaced mode at $306 \mathrm{~cm}^{-1}$, the latter to the combination of the same displaced mode and of the HT active mode predicted at ca. $97 \mathrm{~cm}^{-1}$, see Figure 5. Then the signal peaked at 4361, 4563, and $4687 \mathrm{~cm}^{-1}$ can be assigned to the excitation of the modes predicted at 695,786 , and $864 \mathrm{~cm}^{-1}$, which exhibit the highest HT activity. The last series of comparatively less intense peaks falling between 4986 and $5189 \mathrm{~cm}^{-1}$ can be assigned to the displaced modes 62,80 , and 83, predicted at 1078, 1387, and $1439 \mathrm{~cm}^{-1}$. Of course a deeper analysis, including electronic couplings with the Soret band, is necessary for a better assessment of the $\mathrm{Q}_{y}$ transition.

\section{Conclusions}

The high resolution excitation spectrum of free-base chlorin in the visible region of the spectrum has been analyzed by using a generating function approach together with TD-DFT calculations of the equilibrium geometries and the normal modes of vibrations of the ground electronic state and of the two lowest excited ones.

TD-DFT computations fully support the four orbital model of Gouterman, no $\mathrm{n} \pi^{*}$ excitation being predicted either for the $\mathrm{Q}_{x} \leftarrow \mathrm{S}_{0}$ transition or for the $\mathrm{Q}_{y} \leftarrow \mathrm{S}_{0}$ one. The computed spectrum reproduce all the main features of the observed one. The $\mathrm{Q}_{x} \leftarrow \mathrm{S}_{0}$ band shape is dominated by FC factors, Herzberg-Teller contributions can be safely neglected, but for a few peaks, involving the

modes at 1417 and $1625 \mathrm{~cm}^{-1}$, which exhibit a comparatively higher HT activity that FC one. By contrast, HT effects are significant in the $\mathrm{Q}_{y} \leftarrow \mathrm{S}_{0}$ transition, for which the number of modes show- 
ing a detectable HT activity is higher than those having significant FC factors. The broadness of the $\mathrm{Q}_{y} \leftarrow \mathrm{S}_{0}$ transition is not well reproduced by calculation, being therefore tentatively attributed to an electronic couplings with the Soret bands.

The GF approach has proven to be a very powerful method for the simulation of spectral shapes of large molecules, being able to handle the whole set of normal coordinates, furthermore its computational cost is independent of the value of the temperature, allowing for an easy simulation the temperature dependence of electronic band shapes and radiationless transition rates. ${ }^{37}$ The main limitation of the GF approach, i.e. the lack of the assignment of the simulated spectrum in terms of its vibronic components, can be overcome by an a posteriori analysis of the peaks of interest.

\section{Supporting Information Available}

Mathematical derivation of the working equation Eq. (6). This material is available free of charge via the Internet at http://pubs.acs.org/. 


\section{References}

(1) May, V.; Kühn, O. Charge and Energy Transfer Dynamics in Molecular Systems; WileyVCH: Weinheim, 2004.

(2) O’Regan, B.; Grätzel, M. Nature 1991, 353, 737-740.

(3) Würthner, F.; Archetti, G.; Schmidt, R.; Kuball, H. Angew. Chem., Int. Ed. 2008, 47, 45294532.

(4) Capobianco, A.; Esposito, A.; Caruso, T.; Borbone, F.; Carella, A.; Centore, R.; Peluso, A. Eur. J. Org. Chem. 2012, 2980-2989.

(5) Warshel, A.; Karplus, M. Chem. Phys. Lett. 1972, 17, 7-14.

(6) Domcke, W.; Cederbaum, L. S.; Köppel, H.; von Niessen, W. Mol. Phys. 1977, 34, 17591770.

(7) Kupka, H.; Cribb, P. H. J. Chem. Phys. 1986, 85, 1303.

(8) Peluso, A.; Santoro, F.; Del Re, G. Int. J. Quant. Chem. 1997, 63, 233-244.

(9) Berger, R.; Fischer, C.; Klessinger, M. J. Phys. Chem. A 1998, 102, 7157-7167.

(10) Borrelli, R.; Peluso, A. J. Chem. Phys. 2003, 119, 8437-8448.

(11) Borrelli, R.; Peluso, A. J. Chem. Phys. 2006, 125, 194308-194315.

(12) Borrelli, R.; Peluso, A. J. Chem. Phys. 2008, 128, 044303-7.

(13) Santoro, F.; Improta, R.; Lami, A.; Bloino, J.; Barone, V. J. Chem. Phys. 2007, 126, 084509.

(14) Dierksen, M.; Grimme, S. J. Chem. Phys. 2005, 122, 244101-9.

(15) Hazra, A.; Nooijen, M. Int. J. Quantum Chem. 2003, 95, 643-657.

(16) Toniolo, A.; Persico, M. J. Comput. Chem. 2001, 22, 968-975. 
(17) Jankowiak, H.-C.; Stuber, J. L.; Berger, R. J. Chem. Phys. 2007, 127, 234101-23.

(18) Santoro, F.; Lami, A.; Improta, R.; Bloino, J.; Barone, V. J. Chem. Phys. 2008, 128, 22431117.

(19) Lax, M. J. Chem. Phys. 1952, 20, 1752-1760.

(20) Kubo, R.; Toyozawa, Y. Prog. Theor. Phys. 1955, 13, 160-182.

(21) Mukamel, S.; Sue, J. J. Chem. Phys. 1985, 82, 5291-5292.

(22) Warshel, A.; Chu, Z.; Parson, W. Science 1989, 246, 112-116.

(23) Tang, J.; Lee, M. T.; Lin, S. H. J. Chem. Phys. 2003, 119, 7188-7196.

(24) Ianconescu, R.; Pollak, E. J. Phys. Chem. A 2004, 108, 7778-7784.

(25) Tatchen, J.; Pollak, E. J. Chem. Phys. 2008, 128, 164303-164303-15.

(26) Peng, Q.; Yi, Y.; Shuai, Z.; Shao, J. J. Chem. Phys. 2007, 126, 114302-8.

(27) Peng, Q.; Yi, Y.; Shuai, Z.; Shao, J. J. Am. Chem. Soc. 2007, 129, 9333-9339.

(28) Niu, Y.; Peng, Q.; Deng, C.; Gao, X.; Shuai, Z. J. Phys. Chem. A 2010, 114, 7817-7831.

(29) Peng, Q.; Niu, Y.; Deng, C.; Shuai, Z. Chem. Phys. 2010, 370, 215-222.

(30) Huh, J.; Berger, R. Faraday Discuss. 2011, 150, 363-373.

(31) Lopez, G. V.; Chang, C.-H.; Johnson, P. M.; Hall, G. E.; Sears, T. J.; Markiewicz, B.; Milan, M.; Teslja, A. J. Phys. Chem. A 2012, 116, 6750-6758.

(32) Sharp, T. E.; Rosenstock, K. M. J. Chem. Phys. 1964, 41, 3453-3463.

(33) Kubo, R.; Toyozawa, Y. Prog. Theor. Phys. 1955, 13, 160-182.

(34) Harris, F. Proc. IEEE 1978, 66, 51-83. 
(35) Frisch, M. J.; Trucks, G. W.; Schlegel, H. B.; Scuseria, G. E.; et al., M. A. R. Gaussian 09 Revision A.1, Gaussian Inc. Wallingford CT 2009.

(36) Borrelli, R.; Domcke, W. Chem. Phys. Lett. 2010, 498, 230-234.

(37) Borrelli, R.; Peluso, A. Phys. Chem. Chem. Phys. 2011, 13, 4420.

(38) Reimers, J. R. J. Chem. Phys. 2001, 115, 9103-9109.

(39) Borrelli, R.; Peluso, A. MolFC: A program for Franck-Condon integrals calculation, Package available online at http://www. theochem.unisa.it.

(40) Kemper, M. J. H.; Van Dijk, J. M. F.; Buck, H. M. Chem. Phys. Lett. 1978, 53, 121-124.

(41) Gouterman, M. J. Mol. Spectrosc. 1961, 6, 138-163.

(42) Weiss, C.; Kobayashi, H.; Gouterman, M. J. Mol. Spectrosc. 1965, 16, 415-450.

(43) Dorough, G. D.; Huennekens, F. M. J. Am. Chem. Soc. 1952, 74, 3974-3976.

(44) Keegan, J.; Stolzenberg, A.; Lu, Y.-C.; Linder, R.; Barth, G.; Mocowitz, A.; Bunnerrberg, E.; Djerassi, C. J. Am. Chem. Soc. 1982, 104, 4305.

(45) Keegan, J.; Stolzenberg, A.; Lu, Y.-C.; Linder, R.; Barth, G.; Mocowitz, A.; Bunnerrberg, E.; Djerassi, C. J. Am. Chem. Soc. 1982, 104, 4317.

(46) Huang, W.-Y.; Van Riper, E.; Johnson, L. W. Spectrochim. Acta A 1996, 52, 761-769. 
Table 1: Wavelengths (nm) and oscillator strength of the low-lying electronic states of chlorin at PBE0/6-31+G(d,p) (PBE0/6-311++G**) level of computation.

\begin{tabular}{lccccc}
\hline \hline & \multicolumn{2}{c}{ gas phase } & \multicolumn{3}{c}{ n-Octane } \\
State & $\lambda$ & o. s. & $\lambda$ & o. s. & $\lambda_{\text {exp }}^{a}$ \\
\hline $\mathrm{Q}_{x}$ & $537(541)$ & $0.089(0.094)$ & $543(547)$ & $0.149(0.155)$ & 636 \\
$\mathrm{Q}_{y}$ & $488(491)$ & $0.005(0.004)$ & $490(493)$ & $0.009(0.007)$ & $\geq 490$ \\
$\mathrm{~B}_{x}$ & $354(356)$ & $0.639(0.628)$ & $367(368)$ & $0.951(0.931)$ & 398 \\
$\mathrm{~B}_{y}$ & $351(353)$ & $0.419(0.411)$ & $367(367)$ & $1.237(1.233)$ & 384 \\
\hline \hline
\end{tabular}

${ }^{a}$ from Ref. ${ }^{46}$. 
Table 2: Wavenumbers of the ground $\left(\omega_{g}\right)$ and excited state $\left(\omega_{e}\right)$, equilibrium position displacements (dimensionless), and first derivatives of the electric dipole transition moment (a.u./(§ a.m.u. $\left.{ }^{1 / 2}\right)$ ) for the most active $(|K|>0.1)$ normal modes in the $\mathrm{Q}_{x} \leftarrow \mathrm{S}_{0}$ transition of chlorin. Normal modes with $\left|\mu_{x, y}^{(1)}\right|>0.5$, are also reported, the $\mu_{z}$ component is always negligible.

\begin{tabular}{lrrr|rr}
\hline \hline Mode & $\omega_{e}$ & $\omega_{g}$ & $\mathbf{K}$ & $\mu_{x}^{(1)}$ & $\mu_{y}^{(1)}$ \\
\hline 1 & 24.96 & 21.19 & 0.397 & 0.00 & 0.01 \\
8 & 151.73 & 153.26 & -0.149 & 0.00 & 0.08 \\
12 & 291.10 & 290.19 & 0.126 & 0.00 & -0.05 \\
14 & 304.76 & 312.96 & -0.306 & 0.00 & 0.00 \\
32 & 728.42 & 727.37 & -0.191 & 0.10 & 0.00 \\
35 & 735.81 & 749.66 & -0.122 & 0.00 & 0.00 \\
51 & 901.13 & 919.85 & 0.104 & 0.00 & 0.00 \\
53 & 978.76 & 994.04 & -0.160 & 0.00 & -0.31 \\
74 & 1281.51 & 1278.70 & -0.002 & 0.63 & 0.00 \\
75 & 1297.44 & 1288.38 & -0.110 & 0.00 & -0.08 \\
79 & 1391.05 & 1406.72 & 0.000 & 0.00 & -0.64 \\
80 & 1398.48 & 1416.91 & 0.059 & -0.67 & 0.00 \\
82 & 1416.58 & 1435.11 & 0.137 & 1.15 & 0.00 \\
83 & 1450.82 & 1451.81 & 0.043 & -0.81 & 0.00 \\
85 & 1455.84 & 1469.39 & 0.000 & 0.57 & 0.00 \\
90 & 1527.82 & 1546.56 & 0.000 & 0.00 & -0.54 \\
93 & 1579.91 & 1601.38 & 0.082 & 0.00 & -0.56 \\
96 & 1625.10 & 1673.43 & 0.000 & 1.98 & 0.00 \\
97 & 1640.78 & 1675.00 & -0.087 & 0.00 & -1.00 \\
100 & 3077.55 & 3090.49 & -0.111 & 0.00 & -0.07 \\
\hline \hline
\end{tabular}


Table 3: Wavenumbers of the ground $\left(\omega_{g}\right)$ and excited state $\left(\omega_{e}\right)$, equilibrium position displacements (dimensionless), and first derivatives of the electric dipole transition moment (a.u./( $\AA$ a.m.u. $\left.{ }^{1 / 2}\right)$ ) for the most active $(|K|>0.1)$ normal modes in the $\mathrm{Q}_{y} \leftarrow \mathrm{S}_{0}$ transition of chlorin. Normal modes with $\left|\mu_{x}^{(1)}\right|>2$, are also reported. $\mu_{y, z}^{(1)}$ components are negligible.

\begin{tabular}{lrrr|r}
\hline \hline Mode & $\omega_{e}$ & $\omega_{g}$ & $\mathbf{K}$ & $\mu_{x}^{(1)}$ \\
\hline 1 & 23.81 & 21.19 & 0.340 & -0.03 \\
8 & 150.53 & 153.26 & 0.187 & 0.02 \\
14 & 305.96 & 312.96 & 0.488 & 0.10 \\
62 & 1078.81 & 1077.32 & -0.099 & -1.04 \\
80 & 1387.43 & 1416.91 & -0.192 & -1.76 \\
81 & 1417.53 & 1421.67 & -0.120 & 0.30 \\
83 & 1439.36 & 1451.81 & 0.101 & 1.14 \\
86 & 1451.72 & 1481.91 & -0.108 & -0.87 \\
98 & 1668.11 & 1699.38 & 0.132 & -0.04 \\
2 & 47.42 & 50.90 & 0.000 & 4.73 \\
4 & 96.44 & 97.03 & 0.000 & -4.90 \\
5 & 97.84 & 97.35 & 0.000 & 13.01 \\
6 & 122.80 & 129.19 & 0.000 & 7.08 \\
13 & 301.95 & 308.07 & 0.000 & -13.05 \\
26 & 650.77 & 659.35 & 0.000 & 8.42 \\
28 & 670.43 & 697.73 & 0.000 & 9.81 \\
31 & 695.98 & 713.91 & 0.000 & -26.11 \\
33 & 731.21 & 735.59 & 0.000 & -4.77 \\
35 & 734.65 & 749.66 & 0.000 & 6.26 \\
39 & 773.18 & 790.27 & 0.000 & 10.95 \\
40 & 786.52 & 790.59 & 0.000 & 32.80 \\
44 & 821.47 & 831.02 & 0.000 & -13.26 \\
47 & 848.47 & 875.00 & 0.000 & 12.88 \\
48 & 864.09 & 884.65 & 0.000 & 37.71 \\
102 & 3127.69 & 3133.89 & 0.000 & -2.68 \\
\hline \hline
\end{tabular}




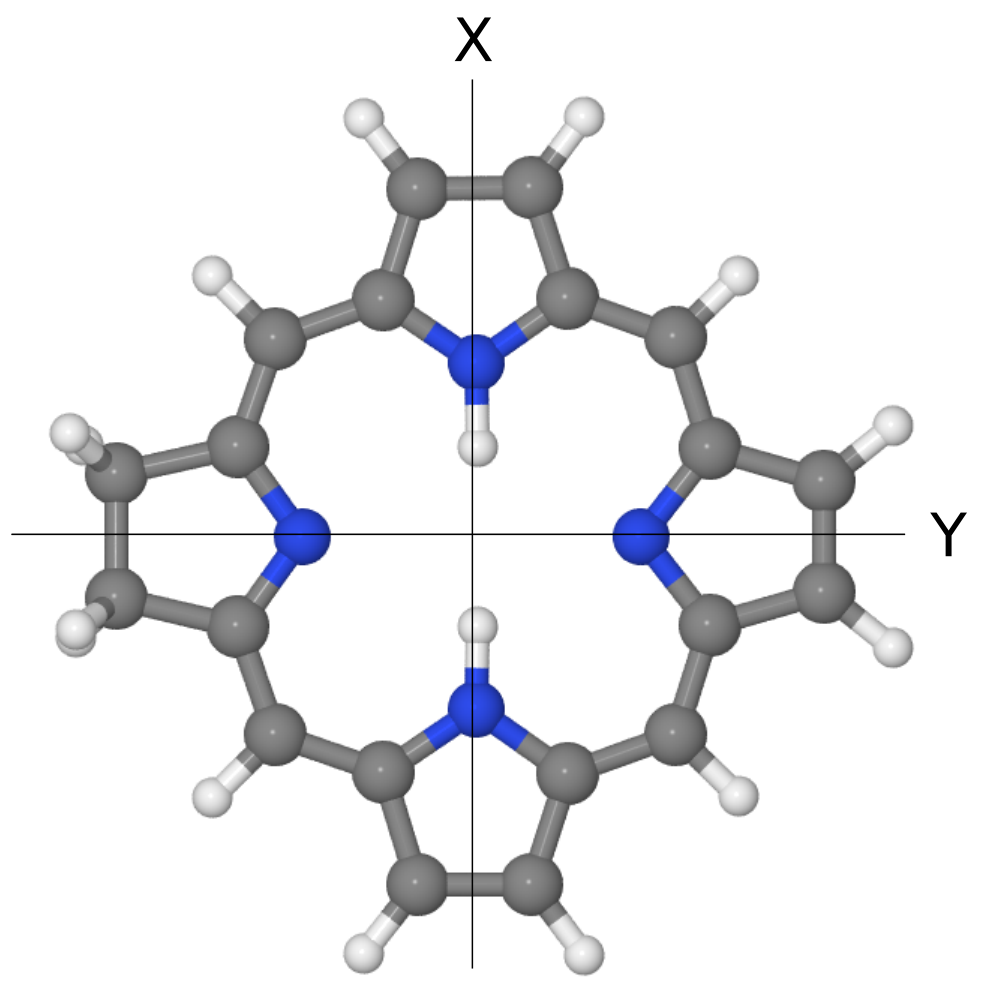

Figure 1: Lowest energy tautomer of free-base chlorin. 


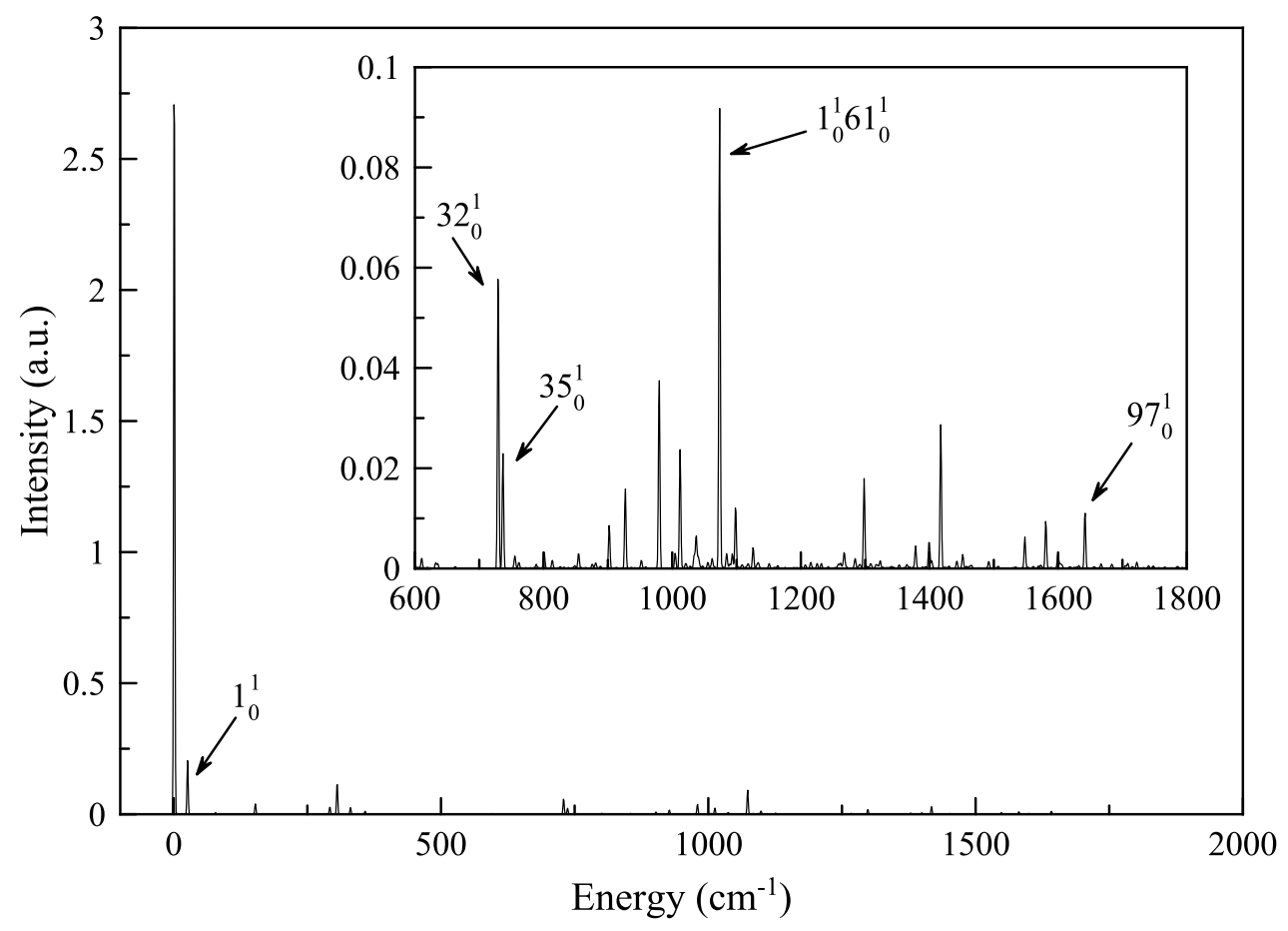

Figure 2: Computed FC spectrum for the $\mathrm{Q}_{x} \leftarrow \mathrm{S}_{0}$ transition of free-base chlorin at 5K. Inset: magnification of the region $600-1800 \mathrm{~cm}^{-1}$.

\section{Graphical TOC Entry}

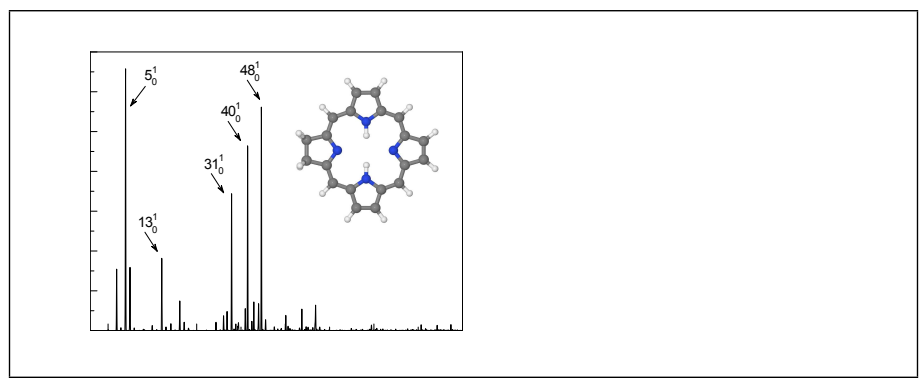




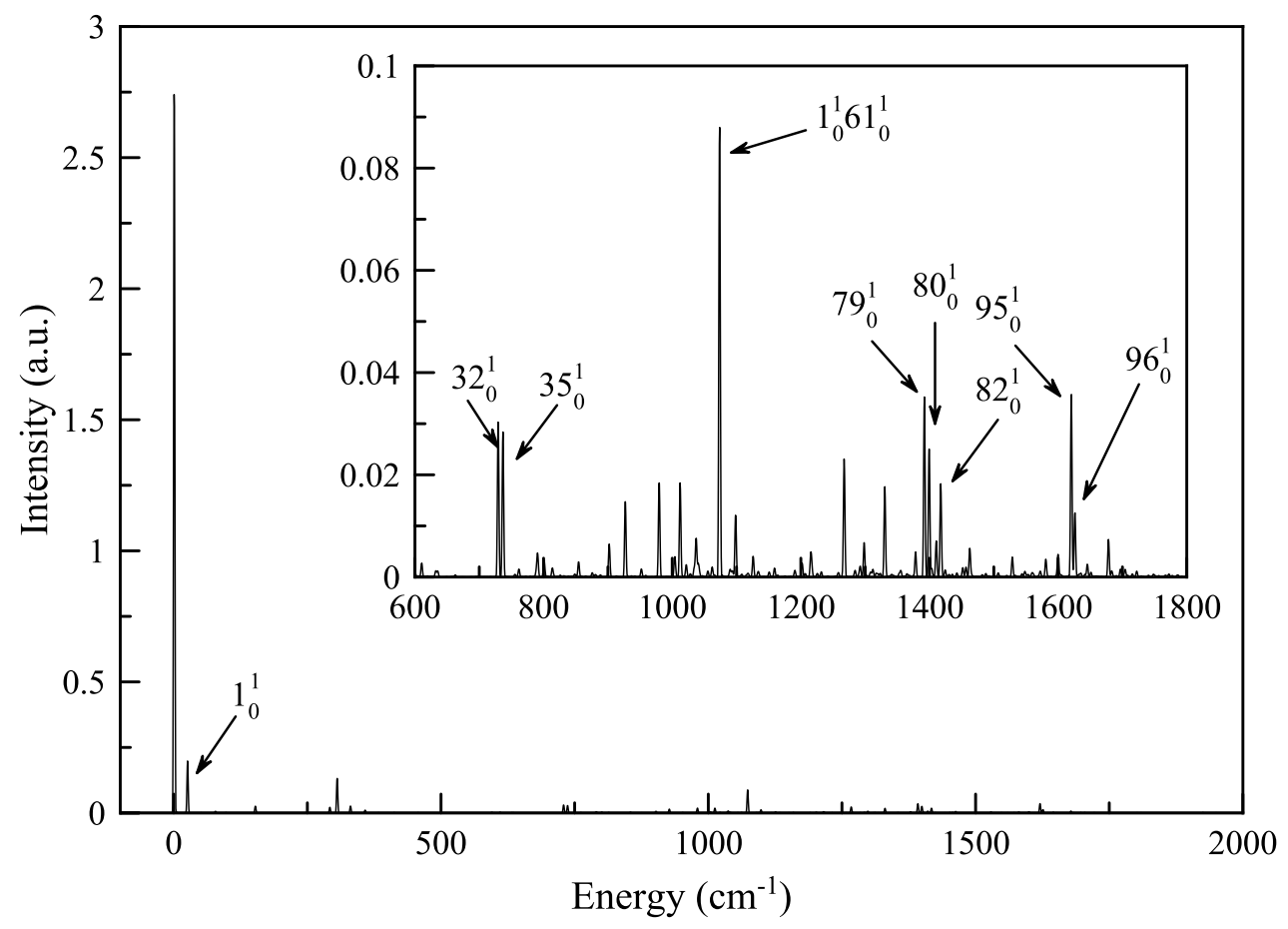

Figure 3: Computed FCHT spectrum for the $\mathrm{Q}_{x} \leftarrow \mathrm{S}_{0}$ transition of chlorin at 5K. Inset: magnification of the region $600-1800 \mathrm{~cm}^{-1}$. 


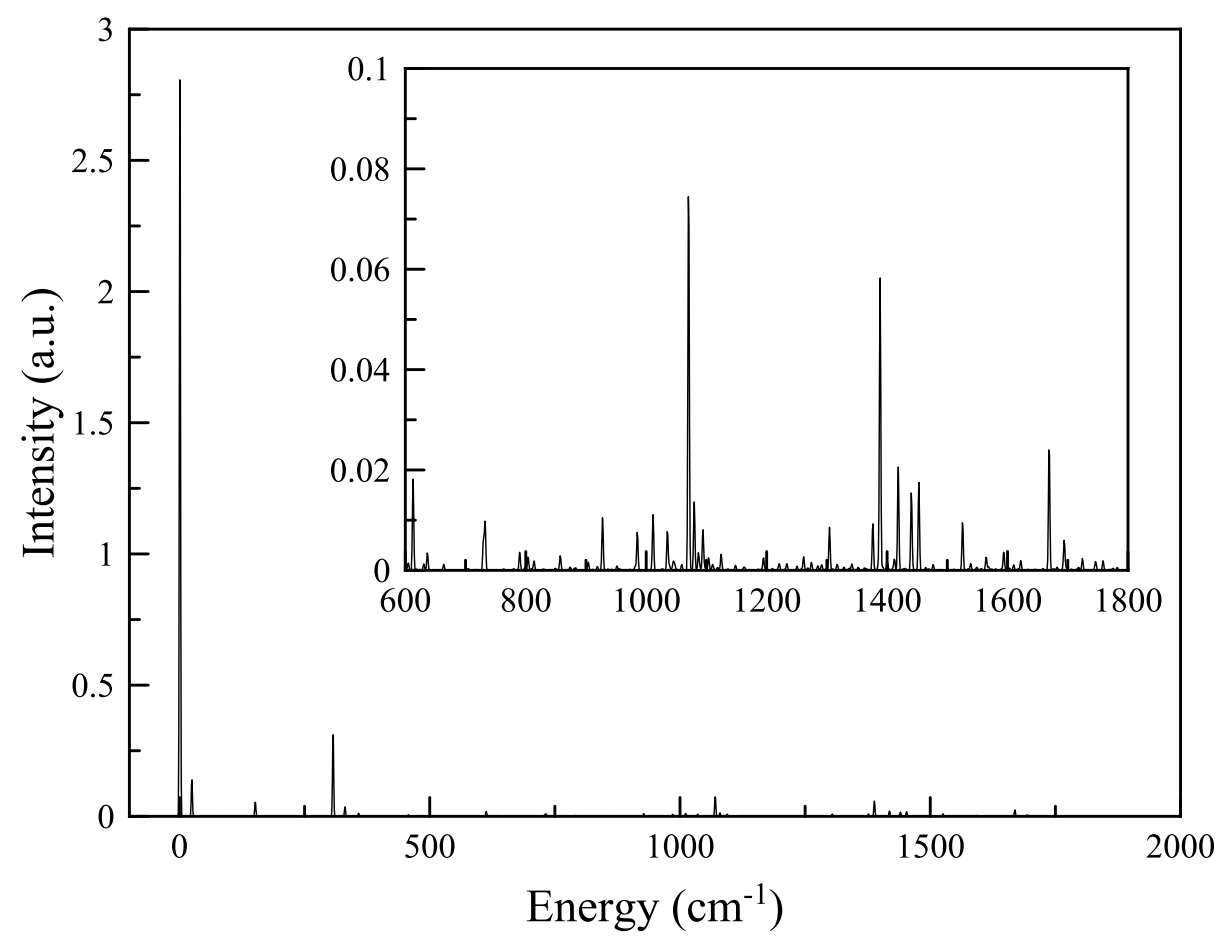

Figure 4: Computed FC spectrum for the $\mathrm{Q}_{y} \leftarrow \mathrm{S}_{0}$ transition of free-base chlorin at $7 \mathrm{~K}$. Inset: magnification of the region $600-1800 \mathrm{~cm}^{-1}$.

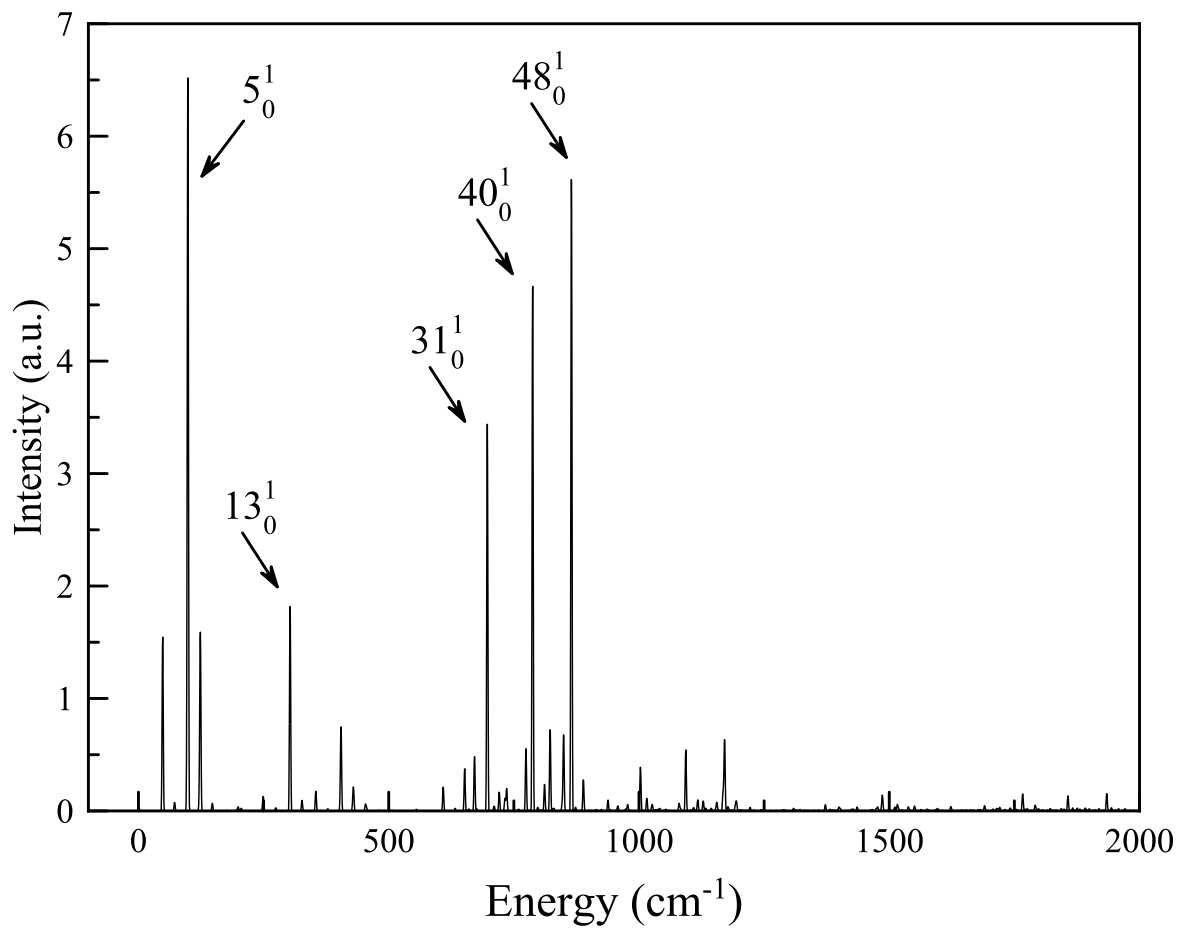

Figure 5: Computed FCHT spectrum for the $\mathrm{Q}_{y} \leftarrow \mathrm{S}_{0}$ transition of free-base chlorin at $7 \mathrm{~K}$. 